\title{
M-CSF in a new biomarker panel with HE4 and CA 125 in the diagnostics of epithelial ovarian cancer patients
}

\author{
Grażyna Ewa Będkowska', Sławomir Ławicki², Ewa Gacuta ${ }^{3}$, Przemysław Pawłowski ${ }^{4}$ and Maciej Szmitkowski ${ }^{2 *}$
}

\begin{abstract}
Background: We investigated plasma levels of M-CSF and conventional tumor markers (HE4 and CA 125) in epithelial ovarian cancer patients as compared to control groups: benign ovarian tumor patients (cysts) and healthy subjects.

Methods: M-CSF levels were determined by ELISA, HE4 and CA 125 levels - by CMIA method.

Results: Our results have demonstrated significant differences in the concentration levels of M-CSF, CA 125 and HE4 between the groups of ovarian cancer patients, cysts patients and the healthy controls. In the groups tested M-CSF demonstrated equal to or higher values than both CA 125 and HE4 in diagnostic sensitivity (SE), positive and negative predictive values (PPV, NPV), and in the area under the ROC curve (AUC), particularly in the group with the serous epithelial sub-type of OC. Moreover, CA 125 showed better results of the aforementioned diagnostic criteria than HE4. The combined use of the parameters studied resulted in a further, significant increase in the value of the diagnostic indicators and in the value of the diagnostic power (AUC), especially in the early stages of ovarian cancer.

Conclusions: These findings suggest a high usefulness of M-CSF in diagnosing the serous sub-type of epithelial ovarian cancer and in discriminating between cancer and non-carcinoma lesions, particularly in new diagnostic panels in combination with CA 125 and HE4 for the detection of EOC in the early stages.
\end{abstract}

Keywords: M-CSF, HE4, CA 125, Epithelial ovarian cancer, Tumor markers

\section{Background}

Epithelial ovarian cancer (EOC) remains the most lethal type of all gynecological cancers despite the development of new treatments and therapies. The malignant tumor of the ovaries occurs at all ages with variation in histological sub-type according to age [1,2]. Established risk factors associated with the increasing prevalence of OC include genetics (BRCA1- breast cancer type 1 susceptibility protein and BRCA2 - breast cancer type 2 susceptibility protein), age (primarily perimenopausal and postmenopausal status), positive familial history (5-10\%), diet (rich in meat and saturated fats), and other reproductive factors $[1,3,4]$. A lack of precise early warning signs is one of the factors that further contributes to the fact that only $25 \%$ of ovarian tumors are identified at

\footnotetext{
*Correspondence: zdb@umwb.edu.pl

${ }^{2}$ Department of Biochemical Diagnostics, Medical University Białystok,

Waszyngtona 15A, Białystok 15-269, Poland

Full list of author information is available at the end of the article
}

a treatable stage I [3]. In the majority of cases $\mathrm{OC}$ is diagnosed in the late stages of the disease when patients have metastatic disease at presentation [2]. Detection at an early stage offers a potential reduction in mortality. Therefore, finding markers which would identify a malignant cell transformation as early as possible is of critical importance [5].

The established tumor markers, such as CA 125 (carbohydrate antigen 125) or human epididymis protein 4 (HE4), can be used in the diagnosis and monitoring of epithelial ovarian cancer [6-8]. A number of researchers and clinicians have been investigating many new diagnostic markers, some of which have recently shown promise, which may be useful in the diagnosis of this type of cancer [7,9]. Different types of substances, for example cytokines such as macrophage-colony stimulating factor (M-CSF), vascular endothelial growth factor (VEGF) and interleukin 10 (IL-10) [10-13], metalloproteinases (MMP-2, MMP-7, MMP-9) and the tissue inhibitor of 
metalloproteinase 1 (TIMP-1) [14-16] or proteomic biomarkers (mesothelin, B7-H4, DcR3, spondin-2) $[17,18]$ are currently being investigated.

Macrophage-colony stimulating factor is one of the cytokines called hematopoietic growth factors (HGFs). M-CSF regulates the growth, differentiation and functionality of neutrophils or macrophages. Additionally, M-CSF has also been implicated in the pathogenesis of cancer disease $[19,20]$. A number of authors focused on the role of M-CSF and its receptor in epithelial malignancies, including those of breast [21-23], lung [24,25], pancreatic [26], cervical [27,28], and ovarian origin [29-31].

The aim of this study was to determine plasma levels of macrophage-colony stimulating factor in comparison to plasma levels of HE4 and the established CA 125 tumor marker in epithelial ovarian cancer (EOC) patients in relation to the control groups: patients with a benign ovarian tumor and healthy subjects. Additionally, comparisons between plasma levels of the parameters tested and cancer stage, its histological sub-type and histological type of benign ovarian tumors were performed. Furthermore, the diagnostic criteria (sensitivity, specificity, positive and negative predictive values) and the receiveroperating characteristic curve (ROC) for the cytokine tested (M-CSF), HE4 and CA 125 alone and in combinations were defined. Moreover, a correlation between the three parameters studied was established.

The data obtained may be used in the evaluation of M-CSF usefulness in diagnosing the stages and histological sub-types of ovarian cancer and in discriminating between ovarian cancer and benign ovarian tumors, especially when analysed with HE4 and CA 125.

\section{Methods}

\section{Patients}

Table 1 shows the characteristics of patients and control groups. The study included 110 epithelial ovarian cancer patients (serous and endometrioid sub-types) diagnosed by the Gynecology Group. The control groups comprised 70 benign ovarian tumor patients (cystis serous or cystis endometrioides) and 50 healthy volunteers. All participants enrolled in the study (cancer and control groups) had postmenopausal status at the time of blood collection. Clinical stages and histological classification based on the criteria of the International Federation of Gynecology and Obstetrics (FIGO) were established in all cases. The ovarian cancer patients and the control group (benign lesions) were treated in the Department of Gynecology, Białystok Medical University Teaching Hospital, Poland, between 2006-2012. Epithelial ovarian cancer and benign ovarian tumor histopathology was established in all cases. Patients with renal failure were excluded from the study due to significantly elevated HE4 concentration levels, indistinguishable from ovarian
Table 1 Characteristics of ovarian cancer patients and control groups: benign ovarian tumor and healthy subjects

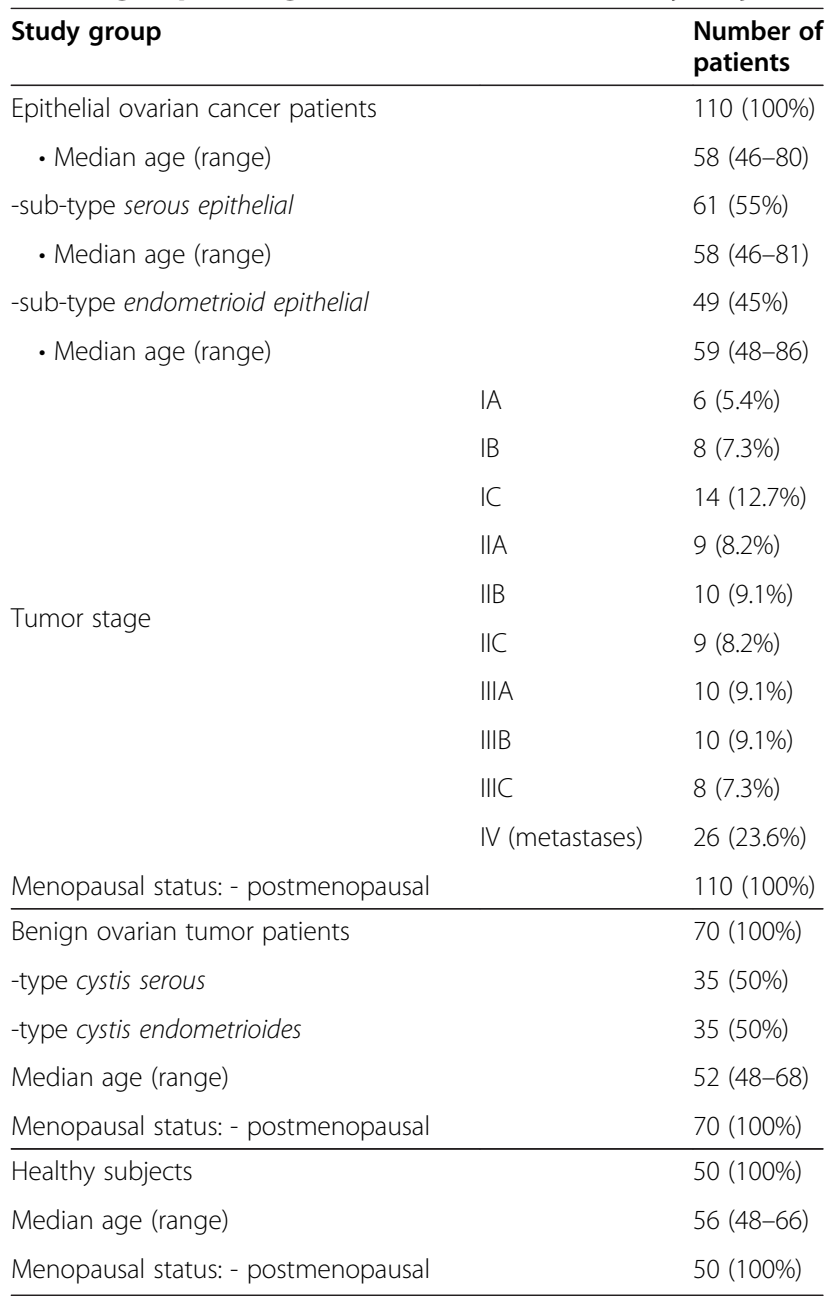

cancer. Written consent including participants' own statements regarding their medical history (i.e. data related to reproductive history, personal or family history of cancer, general health issues - hospitalization or surgery, use of medication) and lifestyle habits including smoking was obtained from all the subjects.

None of the patients had received chemo- or radiotherapy before blood sample collection. Pretreatment staging procedures included physical and blood examinations, ultrasound scanning and chest X-rays. In addition, CT (computed tomography) scans or MRI (magnetic resonance imaging) were performed where necessary.

Healthy patients were recruited from apparently healthy female employees of Białystok Medical University Teaching Hospital between 2006 and 2012. They were not referred from other medical centers. All subjects had undergone annual check-ups (laboratory tests, chest $\mathrm{x}$-ray, cervical cytology screening, mammography). Subjects with a clinical history of prior endometriosis or mild gynecological conditions were excluded. Women included in the control group 
were volunteers who reported no prior history of gynecological conditions and displayed no visible or perceptible changes in the adnexa. The group were examined by a gynecologist prior to blood collection and an ultrasound examination was performed in every case.

The study was approved by the local Ethics Committee of the Medical University in Białystok, numbers: R-I$002 / 314 / 2009$ and R-I-002/262/2010 and all the patients gave their informed consent for the participation in the study.

\section{Biochemical analyses}

Venous blood samples were collected from every patient. Blood was collected into a heparin sodium tube, centrifuged $1000 \mathrm{rpm}$ for $15 \mathrm{~min}$. to obtain plasma samples, and stored at $-85^{\circ} \mathrm{C}$ until assayed. M-CSF was measured with the enzyme-linked immunosorbent assay (ELISA) (Quantikine Human HGFs Immunoassay; R \& D systems, Abingdon, United Kingdom), according to the manufacturer's protocols. Duplicate samples were assessed for each patient. The intra-assay coefficient of variation $(\mathrm{CV} \%)$ of $\mathrm{M}-\mathrm{CSF}-3.4 \%$ at a mean concentration of $227 \mathrm{pg} / \mathrm{ml}$, $\mathrm{SD}=7.7$. The inter-assay coefficient of variation (CV\%) of M-CSF - 3.1\% at a mean concentration of $232 \mathrm{pg} / \mathrm{ml}, \mathrm{SD}=7.3$. The assay showed no significant cross-reactivity or interference with numerous human cytokines and other growth factors.

Plasma concentrations of HE4 and CA 125 were measured by chemiluminescent microparticle immunoassay (CMIA) (Abbott, Chicago, IL, USA). The intra-assay CV for HE4 $-3.7 \%$ at a mean concentration of $39.0 \mathrm{pmol} / \mathrm{L}$, $\mathrm{SD}=1.4$. The inter-assay CV for HE4-2.8\% at a mean concentration of $39.0 \mathrm{pmol} / \mathrm{L}, \mathrm{SD}=1.1$. The intra-assay $\mathrm{CV}$ for CA 125 is reported to be $2.4 \%$ at a mean concentration of $43.5 \mathrm{U} / \mathrm{ml}, \mathrm{SD}=1.1$. The inter-assay $\mathrm{CV}$ for CA 125 is reported to be $3.9 \%$ at a mean concentration of $43.5 \mathrm{U} / \mathrm{ml}, \mathrm{SD}=1.7$.

\section{Statistical analysis}

The statistical analysis was performed using the STATISTICA 8.0 PL program. A preliminary statistical analysis (Chi-square test) revealed that the distribution of cytokine and tumor marker levels did not follow normal distribution. Consequently, nonparametric methods were used to compare levels of the parameters tested between the groups of patients. Comparisons between two groups were performed using the Mann-Whitney test, between multiple groups Kruskal-Wallis tests were calculated with post hoc comparisons according to Dwass-SteeleCritchlow-Fligner method. The ROC analyses were utilized in the evaluation of the diagnostic power of tumor markers and the construction of the curves was performed using GraphRoc Program for Windows. Markers were compared by assessing the significance of differences between the areas under their corresponding ROC curves. The cut-off points of M-CSF $(575.80 \mathrm{pg} / \mathrm{ml})$, HE4 $(75.90 \mathrm{pmol} / \mathrm{L})$ and CA $125(28.40 \mathrm{U} / \mathrm{ml})$ were calculated as 95th percentile from the control group of healthy blood donors.

Data were presented as median and range. Statistically significant differences were defined as comparisons resulting in $\mathrm{p}<0.05$. The Spearman rank correlation was used in the correlation analyses.

\section{Results}

The median of M-CSF levels, similarly to the median levels of the comparative tumor markers HE4 and CA 125 in the total group of $\mathrm{OC}$ and in every stage of advancement (I-IV) of cancer disease were significantly higher when compared to the healthy controls $(\mathrm{p}<0.001$ in all cases) (Table 2). Moreover, plasma concentrations of all tested parameters were significantly higher in more advanced stages (III-IV) than those found in the early stages (I-II) $-\mathrm{p}<0.001$ in all cases.

Patients with ovarian cancer (total group) had statistically considerably higher median levels of the parameters researched ( $\mathrm{p}<0.001$ in all cases) than those observed in the group with benign ovarian tumors (Table 2). We also noticed significantly higher concentrations of M-CSF in II-IV (II - $\mathrm{p}=0.045$; III-IV $-\mathrm{p}<0.001$ ), of HE4 in I-IV $(\mathrm{I}-\mathrm{p}=0.018$; II $-\mathrm{p}=0.001$; III-IV $-\mathrm{p}<0.001)$ and of CA 125 in stages II-IV (II $-\mathrm{p}=0.034$; III-IV $-\mathrm{p}<0.001$ ) of EOC than in the control group with nonmalignant lesions of the ovary.

In the case of the total group with benign ovarian tumors, the concentrations of M-CSF and CA 125 were significantly different than in healthy subjects $(\mathrm{p}<0.001)$.

The analysis according to the histopathological subtypes of EOC revealed statistical differences in the concentrations of M-CSF, HE4 and CA 125 between every cancer sub-type group (serous and endometrioid) and benign tumors control group $(\mathrm{p}<0.001$ in all cases). It was also observed that the distribution of all the tested parameters among two histological subtypes of epithelial ovarian cancer were significantly different $(\mathrm{p}=0.012 ; \mathrm{p}=0.014 ; \mathrm{p}<0.001 ;$ respectively $)$ (Table 2).

Plasma levels of M-CSF and CA 125 in the groups with cystis endometrioides and cystis serous were higher than those in healthy women (in all cases $\mathrm{p}<0.001$ ). Interestingly, the plasma level of HE4 was significantly lower in patients with cystis endometrioides in comparison to the healthy controls $(\mathrm{p}=0.042)$. In addition, we noticed significant differences in the median HE4 $(\mathrm{p}=0.046)$ and CA 125 levels $(\mathrm{p}=0.039)$ when comparing the cystis endometrioides to the cystis serous group (Table 2). 
Table 2 Plasma levels of M-CSF, CA 125 and HE4 in tested groups

\begin{tabular}{|c|c|c|c|c|c|}
\hline & \multicolumn{2}{|l|}{ Groups } & $\begin{array}{l}\text { M-CSF } \\
(\mathrm{pg} / \mathrm{ml})\end{array}$ & $\begin{array}{l}\text { HE4 } \\
\text { (pmol/L) }\end{array}$ & $\begin{array}{l}\text { CA } 125 \\
(\mathrm{U} / \mathrm{ml})\end{array}$ \\
\hline & \multirow{3}{*}{\multicolumn{2}{|c|}{ stage I }} & 1 & $1 / 2$ & 1 \\
\hline \multirow{20}{*}{ Ovarian cancer Median Range } & & & 444.40 & 83.54 & 63.62 \\
\hline & & & $159.80-2702.20$ & $27.50-1093.80$ & $12.50-650.40$ \\
\hline & \multirow{3}{*}{\multicolumn{2}{|c|}{ stage II }} & $1 / 2$ & $1 / 2$ & $1 / 2$ \\
\hline & & & 619.76 & 62.64 & 61.62 \\
\hline & & & 221.70-1764.00 & $24.00-625.10$ & $8.40-998.00$ \\
\hline & \multirow{3}{*}{\multicolumn{2}{|c|}{ stage III }} & $1 / 2 / 4$ & $1 / 2 / 4$ & $1 / 2 / 4$ \\
\hline & & & 706.45 & 117.92 & 766.84 \\
\hline & & & $200.05-3791.05$ & $47.00-1500.00$ & 9.84-2060.78 \\
\hline & \multirow{3}{*}{\multicolumn{2}{|c|}{ stage IV }} & $1 / 2 / 4$ & $1 / 2 / 4$ & $1 / 2 / 4$ \\
\hline & & & 1009.40 & 198.14 & 531.92 \\
\hline & & & 235.64-2091.75 & $36.40-1944.20$ & $13.64-8602.30$ \\
\hline & \multirow{3}{*}{\multicolumn{2}{|c|}{ Total group }} & $1 / 2$ & $1 / 2$ & $1 / 2$ \\
\hline & & & 633.00 & 103.64 & 133.39 \\
\hline & & & 159.80-3791.05 & 24.00-1944.20 & $8.40-8602.30$ \\
\hline & \multirow{3}{*}{\multicolumn{2}{|c|}{ Serous epithelial }} & $1 / 5 / 6 / 7$ & $1 / 5 / 6 / 7$ & $1 / 5 / 6 / 7$ \\
\hline & & & 794.05 & 126.24 & 171.24 \\
\hline & & & $221.7-3791.05$ & $28.60-1944.20$ & $8.40-8602.30$ \\
\hline & \multirow{3}{*}{\multicolumn{2}{|c|}{ Endometrioid epithelial }} & $1 / 6$ & $1 / 6 / 7$ & $1 / 6 / 7$ \\
\hline & & & 606.48 & 68.50 & 114.24 \\
\hline & & & $159.80-3396.20$ & $24.00-1740.00$ & $10.92-1425.00$ \\
\hline \multirow{11}{*}{ Control groups Median Range } & \multirow{9}{*}{ Benign ovarian tumor } & & 3 & 3 & $3 / 8$ \\
\hline & & Cystis endometrioides & 434.28 & 23.18 & 43.44 \\
\hline & & & $125.30-2209.30$ & $14.00-68.60$ & $7.50-2748.00$ \\
\hline & & & 3 & 8 & 3 \\
\hline & & Cystis serous & 468.35 & 43.34 & 20.69 \\
\hline & & & 166.90-1604.40 & 25.40-159.94 & $5.40-451.80$ \\
\hline & & & 3 & & 3 \\
\hline & & Total group & 448.10 & 42.60 & 27.74 \\
\hline & & & $125.30-2209.30$ & 14.00-159.94 & $5.40-2748.00$ \\
\hline & \multirow{2}{*}{\multicolumn{2}{|c|}{ Healthy subjects }} & 298.55 & 44.32 & 10.02 \\
\hline & & & 119.63-1097.00 & $6.20-122.30$ & $5.06-36.60$ \\
\hline
\end{tabular}

1statistically significant when comparing EOC patients with healthy subjects.

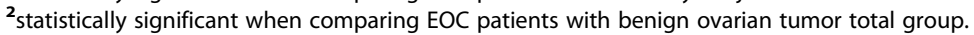

${ }^{3}$ statistically significant when comparing patients with benign ovarian tumor and healthy subjects.

${ }^{4}$ statistically significant when comparing EOC patients in stage III or IV with stage I or II.

${ }^{5}$ statistically significant when comparing EOC patients i.e. sub-type serous with sub-type endometrioid.

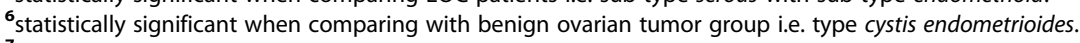

${ }^{7}$ statistically significant when comparing with benign ovarian tumor group i.e. type cystis serous.

${ }^{8}$ statistically significant when comparing patients with benign ovarian tumor i.e. type cystis endometrioides with type cystis serous.

The Spearman's rank correlation was used in the analysis of dependence between the investigated parameters (Additional file 1: the Spearman's rank correlation-results, data not shown). Our analysis revealed positive correlations between the HE4 and CA 125 concentrations in the total group of EOC $(R=0.47, p<0.001)$, between the M-CSF and CA $125(\mathrm{R}=0.4, \mathrm{p}=0.046)$ in patients with stage II cancer as well as between the HE4 and CA 125 levels according to the histopathological sub-types of EOC: endometrioid ( $\mathrm{R}=0.31, \mathrm{p}=0.037)$ or serous $(\mathrm{R}=0.35, \mathrm{p}=0.008)$. Moreover, positive correlations were also observed between the M-CSF and CA 125 levels in the healthy control group $(\mathrm{R}=0.32, \mathrm{p}=$ 0.026). The single negative correlation was obtained for 
the HE4 and CA 125 concentrations $(\mathrm{R}=-0.41, \mathrm{p}=0.036)$ in patients with stage II EOC.

Table 3 shows the diagnostic criteria of parameters tested: sensitivity (SE), specificity (SP), positive predictive value (PPV) or negative predictive value (NPV) in the EOC patients. In the total group of EOC patients MCSF presented the highest diagnostic sensitivity (70\%). HE4 had the highest diagnostic SE in stage I of the disease from among all the parameters tested, although M-CSF demonstrated better results in stages II-IV. We also observed a further increase in the SE value in more advanced cancer stages (with the exception of HE4 in stage II). The combined use of the parameters tested resulted in a dramatic increase in the diagnostic SE for the combination of HE4 + CA 125 in stage I and II, M-CSF + CA 125 in stage III and of M-CSF + HE4 in stage IV of the disease. The maximum range of SE was obtained for the combination of all the parameters tested: in stage I - 80\%, stage II- 92\%, stage III- $96 \%$, IV - 100\% and in the total group of OC - $86 \%$.

In regard to the histopathological sub-types of EOC, we demonstrated the highest range of diagnostic SE for M-CSF in the serous epithelial group and for CA 125 in the endometrioid epithelial group. Similarly, we observed an increase in the SE value in during the combined analysis of two and three parameters for both sub-types of EOC.

M-CSF and HE4 presented the highest diagnostic specificity values (94\%) in every cancer group compared and in every histopathological sub-type of EOC. The combined use of the parameters studied resulted in a decrease in the diagnostic SP (Table 3).

In the total group of EOC patients the PPV had the highest values for HE4 (90\%). HE4 reached the highest values of PPV in stage I in contrast to M-CSF, which reached the highest values in stages II-IV. Similarly, the

Table 3 The diagnostic criteria of M-CSF and in combination with HE4 and CA 125 in epithelial ovarian cancer patients

\begin{tabular}{|c|c|c|c|c|c|c|c|c|}
\hline $\begin{array}{l}\text { Epithelial ovarian } \\
\text { cancer }\end{array}$ & $\begin{array}{l}\text { Diagnostic } \\
\text { criteria (\%) }\end{array}$ & M-CSF & HE4 & CA 125 & M-CSF+ HE4 & M-CSF + CA 125 & HE4+ CA 125 & $\mathrm{M}-\mathrm{CSF}+\mathrm{HE} 4+\mathrm{CA} 125$ \\
\hline \multirow{4}{*}{ stage I } & SE & 40 & 46 & 40 & 64 & 60 & 73 & 80 \\
\hline & SP & 94 & 94 & 92 & 90 & 90 & 88 & 86 \\
\hline & PPV & 87 & 88 & 87 & 86 & 86 & 86 & 85 \\
\hline & NPV & 61 & 63 & 61 & 71 & 69 & 76 & 74 \\
\hline \multirow{4}{*}{ stage II } & SE & 68 & 29 & 63 & 80 & 80 & 81 & 92 \\
\hline & SP & 94 & 94 & 92 & 90 & 90 & 88 & 86 \\
\hline & PPV & 92 & 83 & 89 & 89 & 89 & 87 & 87 \\
\hline & NPV & 75 & 57 & 72 & 82 & 82 & 82 & 91 \\
\hline \multirow{4}{*}{ stage III } & SE & 84 & 66 & 80 & 92 & 94 & 86 & 96 \\
\hline & $\mathrm{SP}$ & 94 & 94 & 92 & 90 & 90 & 88 & 86 \\
\hline & PPV & 93 & 92 & 91 & 90 & 90 & 86 & 87 \\
\hline & NPV & 85 & 73 & 82 & 92 & 94 & 79 & 94 \\
\hline \multirow{4}{*}{ stage IV } & SE & 88 & 81 & 84 & 100 & 96 & 92 & 100 \\
\hline & SP & 94 & 94 & 92 & 90 & 90 & 88 & 86 \\
\hline & PPV & 93 & 93 & 91 & 91 & 91 & 88 & 88 \\
\hline & NPV & 87 & 84 & 85 & 100 & 96 & 92 & 100 \\
\hline \multirow{4}{*}{ Total group } & SE & 70 & 55 & 66 & 84 & 83 & 84 & 86 \\
\hline & SP & 94 & 94 & 92 & 90 & 90 & 88 & 86 \\
\hline & PPV & 81 & 90 & 79 & 89 & 89 & 88 & 86 \\
\hline & NPV & 76 & 68 & 73 & 85 & 84 & 86 & 86 \\
\hline \multirow{4}{*}{ Serous epithelial } & SE & 74 & 70 & 64 & 92 & 82 & 86 & 96 \\
\hline & SP & 94 & 94 & 92 & 90 & 90 & 88 & 86 \\
\hline & PPV & 92 & 92 & 89 & 90 & 80 & 79 & 87 \\
\hline & NPV & 78 & 77 & 73 & 92 & 83 & 86 & 96 \\
\hline \multirow{4}{*}{ Endometrioid epithelial } & SE & 65 & 37 & 71 & 82 & 89 & 82 & 91 \\
\hline & SP & 94 & 94 & 92 & 90 & 90 & 88 & 86 \\
\hline & PPV & 91 & 86 & 90 & 89 & 82 & 87 & 87 \\
\hline & NPV & 73 & 59 & 81 & 83 & 89 & 84 & 92 \\
\hline
\end{tabular}


combined use of the studied markers resulted in a decrease in the PPV values (Table 3). Additionally, we observed the highest PPV value for M-CSF and HE4 in the serous and for M-CSF in the endometrioid sub-types of EOC.

The negative predictive value in the total group of ovarian cancer reached the highest values for M-CSF (76\%). M-CSF also obtained the highest value in stages II-IV with the exception of stage I, where HE4 consistently achieved the best results. Additionally, the NPV values showed a further increase during the combined analysis of the parameters tested (Table 3). The maximum value of NPV was obtained for M-CSF + HE4 and for M-CSF + HE4+ CA 125 in stage IV of the disease although very high values were also observed for the combination of two (69-94\%) and three (74-94\%) biomarkers together in stages I-III of EOC. M-CSF had the highest values in the group with serous and CA 125 in the group with endometrioid sub-types of OC (Table 3).

The relationship between the diagnostic sensitivity and specificity was illustrated by the ROC curve. Table 4 shows the results of our in-depth analysis of the AUC (area under the ROC curve) of every biomarker, alone and in combination, for every stage and for every investigated histological sub-type of the disease. The CA 125 area (0.9277) under the ROC curve is the largest in the total group of EOC patients and its value was marginally higher than that of M-CSF or HE4 (Figures 1 and 2). The AUC of CA 125 was also the largest in the group of patients with stage IV, but interestingly the AUC of MCSF was the largest in the group with stages I-III of the disease. The area under the ROC curve of all the biomarkers tested clearly illustrates an increase in their diagnostic power concurrent with the stage of advancement of the disease (with the exception of HE4 and MCSF in stage IV) (Table 4). Additionally, the combination of all the parameters resulted in an increase in the diagnostic power in every case to the value of: 0.9069 in stage I, 0.9443 in stage II, 0.9714 in stage III and 0.9529 in the total group of EOC. Repeatedly, the largest area under the ROC curve was indicated for the combination of all the aforementioned markers in patients with stage IV cancer. According to histopathological classification, CA125 achieved the best result in endometrioid epithelial (0.9129) and M-CSF in serous epithelial sub-types of ovarian cancer (0.9058). The AUCs of M-CSF and the comparative tumor markers were significantly higher compared to $\mathrm{AUC}=0.5$ in every group of EOC studied (in all cases $\mathrm{p}<0.001$ ).

\section{Discussion}

The search for an effective screening test for ovarian cancer has been the focus of intensive research efforts. Blood flows into and out of tumors and circulates tumor-specific protein profiles, making serum or plasma the ideal biological media for finding a screening biomarker [13]. The co-expression of M-CSF and its transmembrane tyrosine kinase receptor has been detected in epithelial ovarian carcinoma and could be involved in the autocrine growth stimulation of this type of cancer. M-CSF, known as a colony stimulating factor 1 (CSF-1), is also a potent chemoattractant for monocytes, which in turn, can produce factors that stimulate proliferation of ovarian tumor cells including interleukin-1 (IL-1), interleukin-6 (IL-6) and tumor necrosis factor (TNF). Clinical specimens from ovarian cancer metastases display strong immunostaining for both CSF-1 and its receptor in contrast to noninvasive borderline tumors and to benign ovarian tissue [27,29]. HE4 is a novel protein and one of the more promising biomarkers for improving the diagnostic performance in ovarian cancer detection. This is a precursor to the epididymal secretory protein E4. It is overexpressed in ovarian carcinomas, but there is minimal expression in normal ovarian tissue. HE4 promotes migration and adhesion of ovarian cancer cells. It was reported that HE4 could be used as a biomarker for ovarian cancer with a specificity higher than that of CA 125 . This protein was particularly highly expressed in histologic subtypes of serous or endometrioid ovarian carcinoma [6-8].

In this study we investigated the diagnostic usefulness of M-CSF used alone and in combination with HE4 and CA 125 in patients with EOC before surgical intervention or chemo- and/or radiotherapy. Furthermore, we estimated the diagnostic utility of these biomarkers in correlation to the stage and histological type of ovarian cancer.

Our results show that M-CSF as well as HE4 and CA 125 plasma levels in the total group of ovarian cancer patients were statistically significantly higher in comparison to the group of healthy controls. Data regarding MCSF obtained in the present study are also in agreement with our previous studies [10] and with research results of other authors who compared ovarian cancer patients with healthy volunteers, although there were differences in the number and the composition of the groups tested [32-34]. Significantly elevated levels of M-CSF have also been found in the sera of patients with malignancies of the reproductive organs $[32,35,36]$, breast [37] or pancreatic and ampullary cancer [26]. Moreover, it was observed that M-CSF concentrations were statistically different in every group (the analysis related to the stage of advancement of $\mathrm{OC}$ ) compared to the healthy subjects. Similar results for M-CSF were confirmed in our previous investigations [10] and in the studies of other authors [34], although their observations regarding the highest concentrations of M-CSF related to the early stages of the disease and concerned a larger number of sub-types of EOC than our current study (serous, 
Table 4 The diagnostic criteria of the ROC curve for M-CSF and in combination with HE4 and CA 125 in epithelial ovarian cancer patients

\begin{tabular}{|c|c|c|c|c|c|c|c|c|}
\hline Epithelial ovarian cancer & The ROC criteria & M-CSF & HE4 & CA 125 & M-CSF+ HE4 & M-CSF+ CA 125 & HE4 + CA 125 & $\begin{array}{l}\text { M-CSF+ HE4+ } \\
\text { CA } 125\end{array}$ \\
\hline \multirow{4}{*}{ stage I } & $A \cup C$ & $0.7676^{*}$ & $0.7285^{*}$ & $0.7653^{*}$ & $0.8536^{*}$ & $0.8702^{*}$ & $0.8326^{*}$ & $0.9069^{*}$ \\
\hline & SE & 0.0681 & 0.0708 & 0.0416 & 0.0496 & 0.0433 & 0.0538 & 0.0353 \\
\hline & 95\% C.I. & $0.604-0.871$ & $0.590-0.867$ & $0.692-0.794$ & $0.756-0.951$ & $0.785-0.955$ & $0.728-0.938$ & $0.838-0.976$ \\
\hline & $p \mathrm{AUC}=0.5$ & $<0.001$ & 0.0013 & 0.0005 & $<0.001$ & $<0.001$ & $<0.001$ & $<0.001$ \\
\hline \multirow{4}{*}{ stage II } & $A \cup C$ & $0.8662^{*}$ & $0.7647^{*}$ & $0.8653^{*}$ & $0.9008^{*}$ & $0.9113^{*}$ & $0.9208^{*}$ & $0.9443^{*}$ \\
\hline & SE & 0.0483 & 0.0562 & 0.0389 & 0.0387 & 0.0393 & 0.0314 & 0.0265 \\
\hline & 95\% C.I. & $0.772-0.961$ & $0.654-0.876$ & $0.814-0.968$ & $0.825-0.977$ & $0.834-0.988$ & $0.859-0.983$ & $0.892-0.966$ \\
\hline & $p \mathrm{AUC}=0.5$ & $<0.001$ & $<0.001$ & $<0.001$ & $<0.001$ & $<0.001$ & $<0.001$ & $<0.001$ \\
\hline \multirow{4}{*}{ stage III } & AUC & $0.9336^{*}$ & $0.9257^{*}$ & $0.9114^{*}$ & $0.9624^{*}$ & $0.9608^{*}$ & $0.9764^{*}$ & $0.9714^{*}$ \\
\hline & SE & 0.0317 & 0.0291 & 0.0428 & 0.0250 & 0.0302 & 0.0302 & 0.0222 \\
\hline & 95\% C.I. & $0.871-0.996$ & $0.869-0.984$ & $0.828-0.995$ & $0.913-1.011$ & $0.902-1.020$ & $0.903-1.020$ & $0.928-1.015$ \\
\hline & $p A \cup C=0.5$ & $<0.001$ & $<0.001$ & $<0.001$ & $<0.001$ & $<0.001$ & $<0.001$ & $<0.001$ \\
\hline \multirow{4}{*}{ stage IV } & AUC & $0.9096^{*}$ & $0.9125^{*}$ & $0.9474^{*}$ & $0.9872^{*}$ & $0.9665^{*}$ & $0.9422^{*}$ & $0.9894^{*}$ \\
\hline & SE & 0.0414 & 0.0428 & 0.0260 & 0.0086 & 0.0214 & 0.0353 & 0.0083 \\
\hline & 95\% C.I. & $0.829-0.991$ & $0.829-0.996$ & $0.897-0.998$ & $0.970-1.004$ & $0.925-1.008$ & $0.873-1.012$ & 0.973-1.006 \\
\hline & $p \mathrm{AUC}=0.5$ & $<0.001$ & $<0.001$ & $<0.001$ & $<0.001$ & $<0.001$ & $<0.001$ & $<0.001$ \\
\hline \multirow{4}{*}{ Total group } & $A \cup C$ & $0.8562^{*}$ & $0.8324^{*}$ & $0.9277^{*}$ & $0.9257^{*}$ & $0.9271^{*}$ & $0.9182^{*}$ & $0.9529^{*}$ \\
\hline & SE & 0.0316 & 0.0321 & 0.0221 & 0.0212 & 0.0225 & 0.0223 & 0.0172 \\
\hline & 95\% C.I. & $0.794-0.918$ & $0.769-0.895$ & $0.884-0.972$ & $0.884-0.967$ & $0.883-0.971$ & $0.874-0.963$ & $0.919-0.967$ \\
\hline & $p \mathrm{AUC}=0.5$ & $<0.001$ & $<0.001$ & $<0.001$ & $<0.001$ & $<0.001$ & $<0.001$ & $<0.001$ \\
\hline \multirow{4}{*}{ Serous epithelial } & $A \cup C$ & $0.9058^{*}$ & $0.8722^{*}$ & $0.9046^{*}$ & $0.9491^{*}$ & $0.9558^{*}$ & $0.9390^{*}$ & $0.9659^{*}$ \\
\hline & SE & 0.0306 & 0.0347 & 0.0301 & 0.0199 & 0.0199 & 0.0233 & 0.0160 \\
\hline & 95\% C.I. & $0.846-0.966$ & $0.805-0.964$ & $0.846-0.964$ & $0.910-0.989$ & $0.917-0.995$ & $0.893-0.985$ & 0.930-0.997 \\
\hline & $p \mathrm{AUC}=0.5$ & $<0.001$ & $<0.001$ & $<0.001$ & $<0.001$ & $<0.001$ & $<0.001$ & $<0.001$ \\
\hline \multirow{4}{*}{ Endome-trioid epithelial } & $A \cup C$ & $0.7983^{*}$ & $0.7845^{*}$ & $0.9129^{*}$ & $0.8978^{*}$ & $0.8926^{*}$ & $0.8927^{*}$ & $0.9374^{*}$ \\
\hline & SE & 0.0491 & 0.0467 & 0.0305 & 0.0331 & 0.0343 & 0.0330 & 0.0241 \\
\hline & 95\% C.I. & $0.702-0.894$ & $0.694-0.876$ & $0.854-0.973$ & $0.833-0.963$ & $0.825-0.960$ & $0.828-0.958$ & 0.890-0.985 \\
\hline & $p A \cup C=0.5$ & $<0.001$ & $<0.001$ & $<0.001$ & $<0.001$ & $<0.001$ & $<0.001$ & $<0.001$ \\
\hline
\end{tabular}

(C.I. - confidence intervals of AUC).

*statistically significant when comparing tested parameters AUC's with 0.5 AUC.

endometrioid, mucinous, clear cell). It is worth noting that Suzuki and colleagues [38] found no significant difference in M-CSF levels between early and advanced stages of $\mathrm{OC}$, and this is in opposition to the results of our study in which a group of postmenopausal women was studied [10]. It should also be emphasized that MCSF and its receptor gene, c-fms, were expressed in gynecological malignancies, and the co-expression of $\mathrm{M}$-CSF and $c$-fms might be related to progression to the metastatic state [27] and is associated with poor prognosis $[30,38]$. Furthermore, it was observed that 56\% $(14 / 25)$ of the patients with clinically evident OC and normal levels of CA125 had elevated levels of M-CSF [39]. Our results are consistent with the results of other authors who observed increased levels of HE4 and CA
125 in the group of ovarian cancer patients compared to the healthy women group $[8,40]$, although the groups tested were far smaller (60 and 30 women respectively). Similar results were obtained by Molina and others [41] who observed higher concentrations of the aforementioned tumor markers in ovarian (111 patients) as well as in other active gynecological cancers (32 patients) or in different benign gynecological lesions (285 subjects). Furthermore, other researchers have found circulating concentrations of HE4 and CA 125 significantly higher in patients with early and late stages of cancer compared with healthy women, although there were differences in ethnical characteristics of the population selected $[42,43]$. The analysis of research results published by other authors has revealed a finding, almost identical to 


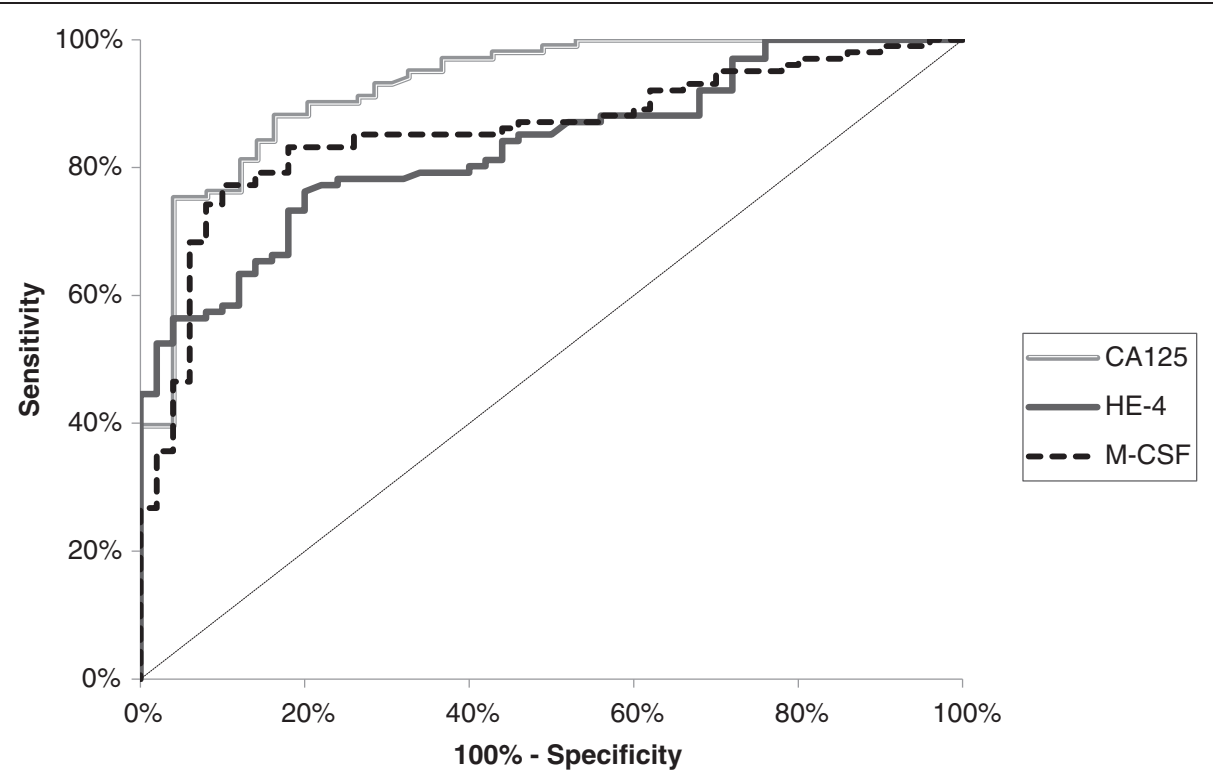

Figure 1 Diagnostic criteria of ROC curve for M-CSF, HE4 and CA 125 in total OC tested group.

ours, regarding significant differences in concentration levels of both tumor markers which were clearly related to the stage of the disease, with significantly higher concentration levels in the advanced stages III-IV than in stages I-II $[41,44]$.

In line with our previous report [10] and others publications $[32,41,45]$ there was a difference in M-CSF concentrations between the EOC patients and the benign (cysts) tumors groups. Gaducci et al. [45] discovered that preoperative serum M-CSF levels were significantly elevated in patients with epithelial OC when compared to those with a benign ovarian disease (cases of serous, mucinous and endometriotic cysts and also of fibromas, thecoma and benign cystic teratomas were analysed). Suzuki et al. [38] confirmed these findings, although malignant germ cell tumors of the ovary and mature cystic teratomas were studied. Furthermore, Burke et al. [46] revealed mRNA expression for M-CSF and its receptor in a majority of malignant and benign ovarian biopsies with minimal to no expression in the normal epithelium. Takagi et al. [32] observed serum M-CSF levels in this type of cancer significantly different from those in patients with a benign ovarian tumor $(p<0.01)$ and with leiomyoma $(\mathrm{p}<0.001)$ as well as between endometrial

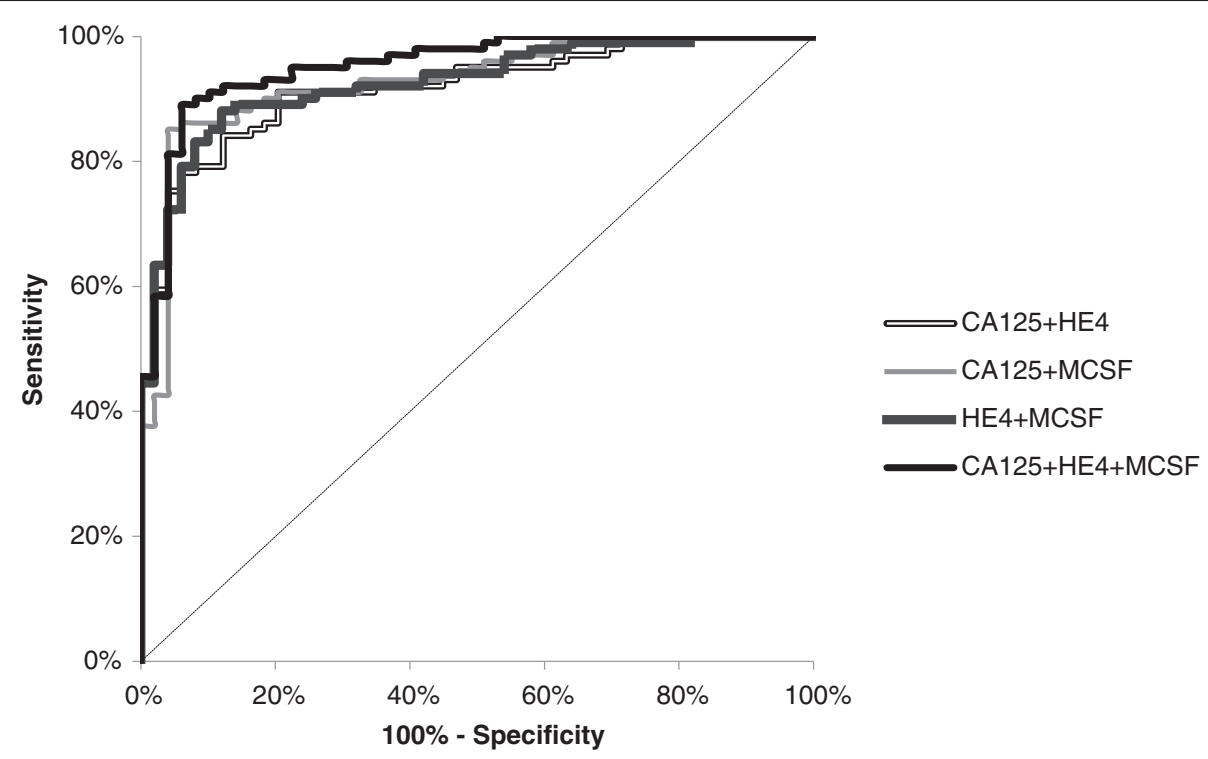

Figure 2 Diagnostic criteria of ROC curve for M-CSF, HE4 and CA 125 in combined analysis in total OC tested group. 
(stages Ib-III) and cervical (stages III-IV) cancer patients and patients with leiomyoma $(\mathrm{p}<0.05)$. Comparable results were obtained in studies conducted on patients with breast malignancies [37]. The findings of the current study regarding comparative tumor markers correspond to the findings reported in the existing literature $[43,47,48]$ and to our previous publications $[10,36]$. Significantly higher serum concentrations of HE4 and CA $125(\mathrm{p}=0.005, \mathrm{p}=0.001, \mathrm{p}<0.0001, \mathrm{p}<0.001$, respectively) $[6,41,49,50]$ were found in OC patients than in those with benign diseases, although there were differences in the menopausal status and composition of the groups compared. In addition, HE4 showed a greater than CA 125, satisfactory ability to distinguish ovarian cancer from endometriosis [51].

In contrast to the results published by Suzuki et al. [29] we observed significantly higher plasma levels of M-CSF in patients with a benign ovarian tumor than in healthy controls. These findings are similar to the results obtained in our previous studies conducted on female patients with uterine myoma [36]. Our present observations concerning both tumor markers are in agreement with published evidence, although HE4 is reported to be less frequently elevated than CA 125 in benign gynecologic disorders $[8,52,53]$. Furthermore, patients with serous epithelial OC displayed significantly different concentrations of all the parameters studied than those with endometrioid epithelial OC. We also demonstrated significant differences in the concentrations of the parameters tested in every group of OC subtypes vs patients with cystis endometrioides and with cystis serous. Comparable results, but only for CA 125 , were published by Nolen et al. [54], although benign cases included a broad spectrum of non-malignant lesions representing a variety of histological origins. Significant differences were also observed, but only for HE4 and CA 125, between the patients with cystis endometrioides and cystis serous. These data are at variance with other publications [28], and may have been partly influenced by study participant selection, although similar results have recently been reported $[7,41,55,56]$. Unfortunately, we could not confirm our findings regarding $\mathrm{M}$-CSF in other publications, since no reports on the subject are available.

The correlation between M-CSF, HE4 and CA 125 levels was estimated by Spearman's rank correlation test and most of the measured values tended to increase for every marker. However, the degree of the correlation was not particularly strong (R: 0.32-0.47), and there were some discordant results. This suggests that each marker was elevated concurrently or under different conditions, and these results also support the necessity of combining the three markers. We found similar results in the existing literature $[8,10,52]$.
In our analysis of postmenopausal subjects, we found that M-CSF alone provided the highest SE, PPV and NPV, (68-88\%, 91-93\%, 75-87\% respectively) of any individual biomarker tested (with the exception of HE4 stage I and CA 125 - EOC endometrioid sub-type). Our results are in agreement with the investigation of Skates et al. [33], whose study group comprised only 60 patients - 50\% fewer than our study group, and with the publications of other authors [57] as well as with our previous studies $[10,58]$. The diagnostic SP was very high and reached the value of $94 \%$ for M-CSF and HE4, and $92 \%$ for CA 125 and these results are in accordance with $[36,59]$ or at variance with [10] our earlier papers and the existing literature $[41,52]$ but they may have been partly influenced by study participant selection.

Nonetheless, a multi-marker approach appears to hold promise for detecting early stage ovarian cancers [6]. Despite a decrease in specificity (86\%) and PPV (85\%), when M-CSF was combined with conventional markers, sensitivity levels improved to $80 \%$ - stage I, to $92-100 \%$ in stages II-IV and to $91 \%$ or $96 \%$ in every histological sub-type of OC. Moreover, in the present study we obtained a better outcome for the diagnostic SE than that attained by Skates et al. [33] and Zhang et al. [34] who found that multiple-marker panels (M-CSF, CA72-4 (carbohydrate antigen 72-4) and CA 125 - 70\%; M-CSF, CA72-4, CA 125 and CA15-3 (carbohydrate antigen $15-3)$ - 71\% respectively) significantly increased preoperative early-stage sensitivity. A comparable increase in the diagnostic SE was previously observed, although it regarded the combined analysis of VEGF with CA 125 and HE4 [59]. Similarly, NPV levels were improved to $74 \%$ - stage I, to $91-100 \%$ in stages II-IV and to $96 \%$ or $92 \%$ in the serous and endometrioid sub-types, substantially higher than NPV levels provided by either marker used alone. These observations are in opposition to the results of other authors who observed distinctly higher values of these diagnostic criteria for HE4 than for CA 125 in premenopausal subjects [60], or higher values of NPV for HE4 or CA 125 alone (72\% and 92\%, respectively) in a group of Asian women [61]. We were unable to compare our findings regarding the aforementioned panel of biomarkers with the findings of other authors, since no reports on the subject are available. The results of the current study support our previous findings regarding the usefulness of M-CSF and CA 125 in the diagnostics of this malignancy [10].

The AUC numerically describes the overall performance of a marker, with the AUC of 1 indicating a perfect SE and SP. We observed that M-CSF had the highest, comparable to conventional markers, diagnostic power in the groups with stages I-III of OC $(0.7676,0.8662$, 0.9336, respectively) and in the patients with serous sub-type of EOC (0.9058). We demonstrated that the 
utilization of a combination of all the markers under investigation consistently provided the highest sensitivity for the detection of every stage, and particularly early stages, of EOC with the AUC value of 0.9069-0.9894, which was superior to the value of either marker used alone. Furthermore, we observed that the areas under the curve for the cytokine tested and the comparative markers were statistically significantly larger compared to $\mathrm{AUC}=0,5$ - borderline of diagnostic usefulness of the test. Test data published by Zhang et al. [34] are at variance with our present results (AUC for: CA 125-0.908, M-CSF - 0.792), although in a few previous studies the AUC values of CA 125 and HE4 for differentiating EOC were $0.82-0.95$ and $0.85-0.96$, respectively $[6,49,50,52,56,62]$ and were significantly higher when the markers were used in combination [50], the results similar to our findings.

\section{Conclusions}

In conclusion, challenges regarding the early diagnosis of epithelial ovarian cancer remain. Although hundreds of biomarkers have been identified as being associated with this common type of cancer, their efficacy in the early diagnosis of the disease has not yet been established. To our knowledge, this is the first study in which plasma levels of M-CSF, HE4 and CA 125 were simultaneously analysed in a relatively large group of untreated patients with primary malignant and nonmalignant ovarian tumors. While each marker has its own strengths and weaknesses, CA 125 still remains better than HE4 in the diagnostics of EOC patients. However, M-CSF obtained even better results than CA 125 . Our findings suggest a highly potential role for M-CSF as a tumor marker in the diagnosis of the serous sub-type of EOC, particularly in combination with HE4 and CA 125 as a new diagnostic panel. The utilization of the tested parameters in combination may also measurably improve the OC diagnosis in the early stages. Additional studies with larger, early-stage carcinoma patient groups may be needed to further strengthen the statistical power of the evidence.

\section{Additional file}

Additional file 1: The Spearman's rank correlation-results.

\footnotetext{
Abbreviations

AUC: Area under ROC curve; BRCA1: Breast cancer type 1 susceptibility protein; BRCA2: Breast cancer type 2 susceptibility protein; CA 125: Carbohydrate antigen 125; CA 15-3: Carbohydrate antigen 15-3; CA 72-4: Carbohydrate antigen 72-4; CEA: Carcinoembryonic antygen; CMIA: Chemiluminescent microparticle immunoassay; CSF-1: Colony stimulating factor 1; CT: Computed tomography; DCR3 ELISA: Enzyme-linked immunosorbent assay; FIGO: International Federation of Gynecology and Obstetrics; HE4: Human epididymis protein 4; HGFs: Hematopoietic growth factors; IL-1: Interleukin 1; IL-6: Interleukin 6; IL-10: Interleukin 10; M-CSF: Macrophage-colony stimulating factor; MMP-2: Metalloproteinase 2;
}

MMP-7: Metalloproteinase 7; MMP-9: Metalloproteinase 9; MRI: Magnetic resonance imaging; OC: Ovarian cancer; PPV: Positive predictive value; NPV: Negative predictive value; ROC: Receiver-operating characteristics; SE: Diagnostic sensitivity; SP: Diagnostic specificity; TNF: Tumor necrosis factor; TIMP-1: Tissue inhibitor of metalloproteinase 1; TNM: Tumor-nodulus-metastasis classification; VEGF: Vascular endothelial growth factor.

\section{Competing interests}

The authors declare that they have no competing interests.

\section{Authors' contributions}

GEB carried out the immunoassays, performed the statistical analysis and drafted the manuscript. St conceived of the study, carried out the immunoassays and helped to draft the manuscript. EG carried out the acquisition of data and participated in the sequence alignment. PP participated in the design and coordination of the study and interpretation of data. MS participated in the design and coordination of the study and interpretation of data. All authors have read and approved the final manuscript.

\section{Authors' information}

This study is a continuation of our research programme concerning cancers of the breast and the reproductive organs, of which several previous manuscripts have been published in journals in the last few years.

\section{Acknowledgements}

This research was financed by a Grant for Scientific Research (nr N N407 530738) in the years 2010-2014 from the Polish Ministry of Science and Higher Education.

\section{Author details}

${ }^{1}$ Department of Haematological Diagnostics, Medical University, Białystok, Poland. 'Department of Biochemical Diagnostics, Medical University Białystok, Waszyngtona 15A, Białystok 15-269, Poland. 'Department of Perinatology, Medical University, Białystok, Poland. ${ }^{4}$ Department of Pediatric Ophthalmology with Squint Treatment Unit, Medical University, Białystok, Poland.

Received: 6 November 2014 Accepted: 22 April 2015

Published online: 03 May 2015

\section{References}

1. Siegel R, Ward E, Brawley O, Jemal A. Cancer statistics: the impact of eliminating socioeconomic and racial disparities on premature cancer deaths. CA Cancer J Clin. 2011;61:212-36.

2. Jemal A, Bray F, Center MM, Ferlay J, Ward E, Forman D. Global cancer statistics. CA Cancer J Clin. 2011;6:69-90.

3. Chetrit A, Hirsh-Yechezkel G, Ben-David Y, Lubin F, Friedman E, Sadetzki S. Effect of BRCA1/2 mutations on long term survival of patients with invasive ovarian cancer: the national Israeli study of ovarian cancer. J Clin Oncol. 2008:26:20-5.

4. Bast Jr RC, Hennessy B, Mills GB. The biology of ovarian cancer: new opportunities for translation. Nat Rev Cancer. 2009;9:415-42.

5. Badgwell D, Bast RC. Early detection of ovarian cancer. Dis Markers. 2007;23:397-410.

6. Hamed EO, Ahmed H, Sedeek OB, Mohammed AM, Abd-Alla AA, Abdel Ghaffar HM. Significance of HE4 estimation in comparison with CA 125 in diagnosis of ovarian cancer and assessment of treatment response. Diagn Pathol. 2013;8:s11.

7. Moore RG, Miller MC, Steinhoff MM, Skates SJ, Lu KH, Lambert-Messerlian G, et al. Serum HE4 levels are less frequently elevated than CA 125 in women with benign gynecologic disorders. Am J Obstet Gynecol. 2012;206:351.e1-8.

8. Azzam AZ, Hashad DI, Kamel NA. Evaluation of HE4 as an extra biomarker to CA 125 to improve detection of ovarian carcinoma: is it time for a step forward? Arch Gynecol Obstet. 2013;288:167-72.

9. Moore RG, Brown AK, Miller MC, Skates S, Allard WJ, Verch T, et al. The use of multiple novel tumor biomarkers for the detection of ovarian carcinoma in patients with a pelvic mass. Gynecol Oncol. 2008;108:402-8.

10. Ławicki S, Gacuta-Szumarska E, Będkowska GE, Szmitkowski M. Hematopoietic cytokines as tumor markers in gynecological malignancies. A multivariate analysis in epithelial ovarian cancer patients. Growth Factors 2012;30:357-66. 
11. Moghaddam MS, Amini A, Morris DL, Pourgholami MH. Significance of vascular endothelial growth factor in growth and peritoneal dissemination of ovarian cancer. Cancer Metastasis Rev. 2012;31:143-62.

12. Liu CZ, Zhang L, Chang XH, Cheng YX, Cheng HY, Ye X, et al. Overexpression and immunosuppressive functions of transforming growth factor 1, vascular endothelial growth factor and interleukin-10 in epithelial ovarian cancer. Chin J Cancer Res. 2012;24:130-7.

13. Kamińska J, Kowalska M, Kotowicz B, Fuksiewicz M. The prognostic value of cytokine levels in patients with cancer. Wspol Onkol. 2006;10:259-62.

14. Schummer M, Drescher C, Forrest R, Gough S, Thorpe J, Hellstrom I, et al. Evaluation of ovarian cancer remission markers HE4, MMP7 and mesothelin by comparison to the established marker CA125. Gynecol Oncol. 2012:125:65-9.

15. Gersthein ES, Kushlinsky DN, Levkina NV, Tereshkina IV, Nosov VB, Laktionov $K P$, et al. Relationship between the expression of VEGF signal components and matrix metalloproteinases in ovarian tumors. Bull Exp Biol Med. 2011;151:449-53.

16. Mahner S, Woelber L, Eulenburg C, Schwarz J, Carney W, Jaenicke F, et al. TIMP-1 and VEGF-165 serum concentration during first-line therapy of ovarian cancer patients. BMC Cancer. 2010;10:139-49.

17. Anderson GL, Mclnthos M, Wu L, Goodman G, Thorpe JD, Bergan L, et al. Assessing lead time of selected ovarian cancer biomarkers: a nested case-control study. J Natl Cancer Inst. 2010;102:26-38.

18. Lin YW, Lin CY, Lai HC, Chiou JY, Chang CC, Yu MH, et al. Plasma proteomic pattern as biomarkers for ovarian cancer. Int J Gynecol Cancer. 2006;16:139-46.

19. Chechlińska M. The role of cytokines in carcinogenesis. J Oncol. 2003;53:648-59.

20. Jasonni VM, Amadori A, Gentile G, Alesi L. Potential role of growth factorsin ovarian cancer. Front Biosci. 1999;1:24-9.

21. Morandi A, Barbetti V, Riverso M, DelloSbarba P, Rovida E. The colony-stimulating factor-1 (CSF-1) receptor sustains ERK1/2 activation and proliferation in breast cancer cell lines. PLoS One. 2011;6(11):e27450.

22. Ławicki S, Będkowska GE, Wojtukiewicz M, Szmitkowski M. Hematopoietic cytokines as tumor markers in breast malignancies. A multivariate analysis with ROC curve in breast cancer patients. Adv Med Sci. 2013;58:207-15.

23. Toy EP, Bonafé N, Savlu A, Zeiss C, Zheng W, Flick M, et al. Correlation of tumor phenotype with c-fms proto-oncogene expression in an in vivo intraperitoneal model for experimental human breast cancer metastasis. Clin Exp Metastasis. 2005;22:1-9.

24. Bahar B, Cayci B, Coskun U, Buyukberber S, Benekli M, Yildiz R. Granulocyte colony stimulating factor (G-CSF) and macrophage colony stimulating factor (M-CSF) as potential tumor markers in non-small cell lung cancer diagnosis. Asian Pacific J Cancer Prev. 2010;11:709-12.

25. Kamińska J, Kowalska M, Kotowicz B. Pretreatment serum levels of cytokines and cytokine receptors in patients with non-small cell lung cancer, and correlations with clinicopathological features and prognosis. Oncology. 2006;70:115-25.

26. Vasiliades G, Kopanakis N, Vasiloglou M, Zografos G, Margaris H, Masselou K, et al. Role of the hematopoietic cytokines SCF, IL-3, GM-CSF and M-CSF in the diagnosis of pancreatic and ampullary cancer. Int J Biol Markers. 2012;27:186-94.

27. Baiocchi G, Kavanagh JJ, Talpaz M, Wharton JT, Gutterman JU, Kurzrock R. Expression of the macrophage colony-stimulating factor and its receptor in gynecologic malignancies. Cancer. 1991;67:990-6.

28. Kirma N, Hammes LS, Liu YG, Nair HB, Valente PT, Kumar S, et al. Elevated expression of the oncogene $\mathrm{c}$-fms and its ligand, the macrophage colonystimulating factor-1, in cervical cancer and the role of transforming growth factor-beta1 in inducing c-fms expression. Cancer Res. 2007;67:1918-26.

29. Suzuki M, Ohwada M, Aida I, Tamada T, Hanamura T, Nagatomo M. Macrophage colony - stimulating factor as a tumor marker for epithelial ovarian cancer. Obstet Gynecol. 1993;82:946-50.

30. Chambers SK, Kacinski BM, Ivins CM, Carcangiu ML. Overexpression of epithelial macrophage colony-stimulating factor (CSF-1) and CSF-1 receptor: a poor prognostic factor in epithelial ovarian cancer, contrasted with a protective effect of stromal CSF-1. Clin Cancer Res. 1997;3:999-1007.

31. Haaften-Day C, Yu S, Fengji X, Yinhua Y, Berchuck A, Havrilesky LJ, et al. OVX1, macrophage-colony stimulating factor, and CA-125-II as tumor markers for epithelial ovarian carcinoma. Cancer. 2001;92:2837-44.

32. Takagi A, Takeda S, Matsuoka K, Kinoshita K, Shimizu K. Macrophage colony stimulating factor (M-CSF) production in vivo and in vitro in gynecologic malignancies. Int J Clin Oncol. 1999:4:142-7.
33. Skates S, Horick N, Yu Y, Xu FJ, Berchuck A, Havrilesky LJ, et al. Preoperative sensitivity and specificity for early-stage ovarian cancer when combining cancer antigen CA 125 II, CA 15-3, CA 72-4, and macrophage colonystimulating factor using mixtures of multivariate normal distributions. J Clin Oncol. 2004;22:4059-66.

34. Zhang Z, Yu Y, Xu FX, Berchuck A, Haaften-Day C, Havrilesky L, et al. Combining multiple serum tumor markers improves detection of stage I epithelial ovarian cancer. Gynecol Oncol. 2007;107:526-31.

35. Ławicki S, Będkowska E, Gacuta- Szumarska E, Knapp P, Szmitowski M. The plasma levels and diagnostics utility of stem cell factor (SCF) and macrophage-colony stimulating factor (M-CSF) in cervical cancer patients. Pol Merkur Lek. 2008;145:38-42.

36. Ławicki S, Będkowska GE, Gacuta-Szumarska E, Szmitkowski M. Hematopoietic cytokines as tumor markers in gynecological malignancies: a multivariate analysis with ROC curve in endometrial cancer patients. Growth Factors. 2012;30:29-36.

37. Ławicki S, Będkowska GE, Szmitkowski M. VEGF, M-CSF and CA 15-3 as a new tumor marker panel in breast malignancies: a multivariate analysis with ROC curve. Growth Factors. 2013:31:98-105.

38. Suzuki M, Kobajashi H, Ohwada M, Terao T, Sato I. Macrophage colonystimulating factor as a marker for malignant germ cell tumors of the ovary. Gynecol Oncol. 1998;68:35-7.

39. Xu FJ, Ramakrishnan SD, Daly L, Soper JT, Berchuk A, Clarke-Pearson DL, et al. Increased serum levels of macrophage colony-stimulating factor in ovarian cancer. Am J Obstet Gynecol. 1991;165:1356-62.

40. Ghasemi N, Ghobadzadeh S, Zahraei M, Mohammadpour H, Bahrami S, Ganje MB, et al. HE4 combined with CA125: favorable screening tool for ovarian cancer. Med Oncol. 2014;31:808.

41. Molina R, Escudero JM, Auge JM, Filella X, Foj L, Torne A, et al. HE4 a novel tumour marker for ovarian cancer: comparison with CA 125 and ROMA algorithm in patients with gynaecological diseases. Tumor Biol. 2011;32:1087-95.

42. Yurkowetsky Z, Skates S, Lomakin A, Nolen B, Pulsipher T, Modugno F, et al. Development of a multimarker assay for early detection of ovarian cancer. J Clin Oncol. 2010;28:2159-66.

43. Kim YM, Whang DH, Park J, Kim SH, Lee SW, Park HA, et al. Evaluation of the accuracy of serum human epididymis protein 4 in combination with CA125 for detecting ovarian cancer: a prospective case-control study in a Korean population. Clin Chem Lab Med. 2011;49:527-34

44. Cooper BC, Sood AK, Davis CS, Ritchie JM, Sorosky Jl, Anderson B, et al. Preoperative CA125 levels: an independent prognostic factor for epithelial ovarian cancer. Obstet Gynecol. 2002;100:59-64.

45. Gadducci A, Ferdeghini M, Castellani C, Annicchiarico C, Prontera C, Facchini $V$, et al. Serum macrophage colony stimulating factor (M-CSF) levels in patients with epithelial ovarian cancer. Gynecol Oncol. 1998;70:111-4.

46. Burke F, Relf M, Negus R, Balkwill F. A cytokine profile of normal and malignant ovary. Cytokine. 1996;8:578-85.

47. Chudecka-Głaz A, Rzepka-Górska I, Wojciechowska I. Human epididymal protein 4 (HE4) is a novel biomarker and a promising prognostic factor in ovarian cancer patients. Eur J Gynaecol Oncol. 2012;33:382-90.

48. Nolen B, Velikokhatnaya L, Marrangoni A, De Geest K, Lomakin A, Bast Jr RC, et al. Serum biomarker panels for the discrimination of benign from malignant cases in patients with an adnexal mass. Gynecol Oncol. 2010;117:440-5.

49. Ruggeri G, Bandiera E, Zanotti L, Belloni S, Ravaggi A, Romani C, et al. HE4 and epithelial ovarian cancer: comparison and clinical evaluation of two immunoassays and a combination algorithm. Clin Chim Acta. 2011:412:1447-53.

50. Zheng H, Gao Y. Serum HE4 as a useful biomarker in discriminating ovarian cancer from benign pelvic disease. Int J Gynecol Cancer. 2012;22:1000-5.

51. Kadija S, Stefanovic A, Jeremic K, Radojevic MM, Nikolic L, Markovic I, et al, The utility of human epididymal protein 4 , cancer antigen 125 , and risk of malignancy algorithm in ovarian cancer and endometriosis. Int J Gynecol Cancer. 2012;22:238-44.

52. Park Y, Lee J-H, Hong DJ, Lee EY, Kim H-S. Diagnostic performances of HE4 and CA125 for the detection of ovarian cancer from patients with various gynecologic and non-gynecologic diseases. Clin Biochem. 2011;44:884-8.

53. Gorelik E, Landsittel DP, Marrangoni AM, Modugno F, Veikokhatnaya L, Winans MT, et al. Multiplexed immunobead-based cytokine profiling for early detection of ovarian cancer. Cancer Epidemiol Biomark \& Prev. 2005;14:981-7. 
54. Nolen B, Marrangoni A, Velikokhatnaya L, Prosser D, Winans M, Gorelik E, et al. A serum analysis of ovarian epithelial tumor genesis. Gynecol Oncol. 2009; 112:47-54.

55. Drapkin R, von Horsten HH, Lin Y, Mok SC, Crum CP, Welch WR, et al. Human epididymis protein 4 (HE4) is a secreted glycoprotein that is overexpressed by serous and endometrioid ovarian carcinomas. Cancer Res. 2005;65:2162-9.

56. Macuks R, Baidekalna I, Donina S. An ovarian cancer malignancy risk index composed of HE4, CA125, ultrasonographic score, and menopausal status: use in differentiation of ovarian cancers and benign lesions. Tumor Biol. 2012;33:1811-7.

57. Bodnar L, Wcisło G, Miedzińska-Maciejewska M, Szczylik C. The role of macrophage colony-stimulating factor (M-CSF) in prognosis, diagnosis and in the treatment of epithelial ovarian cancer. Wspol Onkol. 2003;7:412-9.

58. Ławicki S, Będkowska GE, Gacuta-Szumarska E, Czygier M, Szmitkowski M The plasma levels and diagnostic utility of selected hematopoietic growth factors in endometrial cancer patients and with myoma uteri. Pol Merkur Lek. 2010;167:354-8.

59. Ławicki S, Będkowska GE, Gacuta-Szumarska E, Szmitkowski M. The plasma concentration of VEGF, HE4 and CA125 as a new biomarkers panel in different stages and sub-types of epithelial ovarian tumors. J Ovarian Res. 2013;6:45-55.

60. Holcomb K, Vucetic Z, Miller MC, Knapp RC. Human epididymis protein 4 offers superior specificity in the differentiation of benign and malignant adnexal masses in premenopausal women. Am J Obstet Gynecol. 2011;205:358.e1-6.

61. Chan KKL, Chen CA, Nam JH, Ochiai K, Wilailak S, Choon AT, et al. The use of HE4 in the prediction of ovarian cancer in Asian women with a pelvic mass. Gynecol Oncol. 2013;128:239-44.

62. Sandri MT, Bottari F, Franchi D, Boveri S, Candiani M, Ronzoni S, et al. Comparison of HE4, CA 125 and ROMA algorithm in women with a pelvic mass: correlation with pathological outcome. Gynecol Oncol. 2013;128:233-8

\section{Submit your next manuscript to BioMed Central and take full advantage of:}

- Convenient online submission

- Thorough peer review

- No space constraints or color figure charges

- Immediate publication on acceptance

- Inclusion in PubMed, CAS, Scopus and Google Scholar

- Research which is freely available for redistribution 